\section{Aging of human hematopoietic stem cells is linked to changes in Cdc42 activity}

\author{
Amanda Amoah, ${ }^{1}$ Anja Keller, ${ }^{1}$ Ramiz Emini, ${ }^{2}$ Markus Hoenicka, ${ }^{2}$ Andreas \\ Liebold, ${ }^{2}$ Angelika Vollmer, ${ }^{1}$ Karina Eiwen, ${ }^{1}$ Karin Soller, ${ }^{1}$ Vadim Sakk, ${ }^{1}$ Yi \\ Zheng, ${ }^{3}$ Maria Carolina Florian ${ }^{1}{ }^{\circ}$ and Hartmut Geiger ${ }^{1}$
}

${ }^{1}$ Institute of Molecular Medicine, Ulm University, UIm, Germany; ${ }^{2}$ Department of Cardiothoracic and Vascular Surgery, UIm University Hospital, Ulm, Germany and ${ }^{3}$ Cincinnati Children's Hospital Medical Center and University of Cincinnati, Cincinnati, $\mathrm{OH}$, USA

${ }^{\circ} A K$ current address: Zentrum für Integrative Psychiatrie, Lübeck Campus, Lübeck, Germany

${ }^{\circ}$ RE current address: Herzzentrum, Uniklinik Köln, Köln, Germany

${ }^{\circ}$ MCF current address: Bellvitge Institute for Biomedical Research, IDIBELL, Barcelona, Spain

\section{ABSTRACT}

T $\mathrm{n}$ this study, we characterize age-related phenotypes of human hematopoietic stem cells (HSC). We report increased frequencies of HSC, hematopoietic progenitor cells and lineage negative cells in the elderly but a decreased frequency of multi-lymphoid progenitors. Aged human HSC further exhibited a delay in initiating division ex vivo though without changes in their division kinetics. The activity of the small RhoGTPase Cdc42 was elevated in aged human hematopoietic cells and we identified a positive correlation between Cdc42 activity and the frequency of HSC upon aging. The frequency of human HSC polar for polarity proteins was, similar to the mouse, decreased upon aging, while inhibition of Cdc42 activity via the specific pharmacological inhibitor of Cdc42 activity, CASIN, resulted in re-polarization of aged human HSC with respect to Cdc42. Elevated activity of Cdc42 in aged HSC thus contributed to age-related changes in HSC. Xenotransplant, using NBSGW mice as recipients, showed elevated chimerism in recipients of aged compared to young HSC. Aged HSC treated with CASIN ex vivo displayed an engraftment profile similar to recipients of young HSC. Taken together, our work reveals strong evidence for a role of elevated Cdc42 activity in driving aging of human HSC, and similar to mice, this presents a likely possibility for attenuation of aging in human HSC.

\section{Introduction}

Aging is associated with tissue degeneration, aging-related diseases and an increased susceptibility to infections. ${ }^{1,2}$ These hallmarks of aging have been linked to aging-related changes within somatic stem cell compartments, and primarily investigated in animal models like mice. ${ }^{3,4}$ One of the most extensively studied somatic stem cell-based system is the hematopoietic system. Hematopoietic stem cells (HSC) maintain blood homeostasis and show an age-related decline in overall function in mice, ${ }^{5}$ which includes an increase in myelopoiesis, ${ }^{6}$ accumulation of DNA damage, ${ }^{7}$ changes in epigenomic and transcriptional programs, ${ }^{8}$ decreased cell polarity and aberrant activity of the small RhoGTPase Cdc42. ${ }^{9}$ Although significant progress has been achieved in elucidating mechanisms of aging of murine HSC, it remains unclear whether these mechanisms can be simply extrapolated to other species, including humans. Early studies on HSC number and function in larger mammals showed, based on stochastic modeling, clear differences in HSC biology and aging to the murine model..$^{10,11}$ These data suggested that HSC from non-human primates cycle more slowly and that fewer numbers of HSC clones actively contribute to hematopoiesis in humans at steady-state than in mice. This connotes that non-human primates and murine HSC may undergo different stress intensities such as the rate of accruing DNA damage and, as a result, may exhibit dissimilarities in aging.
Ferrata Storti Foundation

Haematologica 2022

Volume 107(2):393-402

\section{Correspondence:}

HARTMUT GEIGER,

hartmut.geiger@uni-ulm.de

Received: August 13, 2020.

Accepted: December 22, 2020.

Pre-published: January 14, 2021.

https://doi.org/10.3324/haematol.2020.269670

(C2022 Ferrata Storti Foundation

Material published in Haematologica is covered by copyright. All rights are reserved to the Ferrata Storti Foundation. Use of published material is allowed under the following terms and conditions:

https://creativecommons.org/licenses/by-nc/4.0/legalcode. Copies of published material are allowed for personal or internal use. Sharing published material for non-commercial purposes is subject to the following conditions:

https://creativecommons.org/licenses/by-nc/4.0/legalcode, sect. 3. Reproducing and sharing published material for commercial purposes is not allowed without permission in writing from the publisher. 
In addition, mice show an increase in HSC frequency with age, while the rhesus monkey, shows a decrease with age. ${ }^{12}$ Moreover, even among distinct murine inbred strains, HSC number and function is distinct upon aging, like C57BL/6 mice present with an elevated number of HSC upon aging, but not so in DBA/2 animals. ${ }^{13,14}$ For these reasons, novel studies into understanding mechanisms of aging of human HSC are warranted and are a prerequisite to bolster the transition of this knowledge into the clinic.

Age-related changes in the frequency and function of HSPC have been in part previously described by a small number of groups. One study for example reported no changes in the re-population potential of aged HSC and a decreased propensity for myeloid differentiation while another recorded a decline in the reconstitution capacity of aged HSC with an increased myeloid differentiation potential. ${ }^{15,16}$ Both groups used NOD.Cg-Prkdssid $I l 2 \mathrm{rg}^{\mathrm{tm} / W_{i} /} / \mathrm{SzJ}$ (NSG) animals as recipients for in vivo xenotransplantation to study the function of human HSC. This model requires irradiation of the recipient animal for the successful establishment of xenotransplants ${ }^{17}$ which might contribute to variable secondary effects not linked to the transplanted HSC. New and improved mouse models have been created that do not require pre-conditioning of the recipients for achieving human xenochimerism. ${ }^{18,19}$ NOD.Cg-Kit ${ }^{W-41]}$ Tyr ${ }^{+}$Prkds sid $I / 2 \mathrm{rg}^{\mathrm{tm} / W_{i} /} / \mathrm{ThomJ}$ (NBSGW) animals bear in addition to the NSG genotype a mutation in the Kit gene. ${ }^{19}$ The Kit mutation enables donor cells to efficiently engraft without irradiation. ${ }^{20}$ We characterize here aged human HSC with a special focus on likely shared hallmarks of age-related changes among human and murine HSC and describe a novel approach to attenuate aging of human HSC. Our data support the possibility of rejuvenating the function of aged human HSC due to similarities between aging of murine and human HSC.

\section{Methods}

\section{Primary cells}

Bone marrow cells were isolated from young (range, 23-39 years; median age 27 years) donors acquired from Cincinnati Children's Hospital Medical Center and aged (range, 58-82 years; median age 66 years) individuals undergoing heart surgery at the Ulm University Clinic, Department of Heart, Thoracic and Vascular Surgery (additional details of age strata are provided in the Online Supplementary Methods). All donors were hematologically healthy. Sample collection and investigation was approved by the Internal Review Board (Ethikkomission) of Ulm University (392/16).

\section{Flow cytometric analysis and cell sorting}

Mononuclear cells (MNC) were thawed and stained in phosphate-buffered saline (PBS) supplemented with $3 \%$ fetal bovine serum (FBS) with human specific antibodies (see the Online Supplementary Methods for details). Different cell populations were identified and sorted on a BD FACS ARIA II 4L SORP (BD Biosciences) according to the markers used by van Galen et al. ${ }^{21}$

\section{Single cell division assay}

Single HSC were sorted into Terasaki plates and checked every 12 hours under a light microscope (further details are provided in the Online Supplementary Methods).

\section{Colony forming unit assay}

In order to assess the myeloid and erythroid generative potential of samples, 200 HSC were seeded in methylcellulose medium (further details are provided in the Online Supplementary Methods).

\section{Xenotransplantation}

All animal experiments were carried out in accordance to institutional guidelines and approved by the Regierungspräsidium Tübingen (TVA 1412). Five hundred HSC were injected via the tail vein into non-conditioned or low dose irradiated (1.6 Gy) NBSGW mice. At 8 and 12 weeks, aspirates were drawn from the bone marrow (BM) of mice after administering anesthesia. Human cells were identified using human-specific antibodies (see the Online Supplementary Methods for further details) and analyzed on LSR Fortessa SORP flow cytometer (BD Biosciences). Human chimerism was determined as a percentage of total CD $45+$ cells and mature cells, as a percentage of human CD $45+$ cells.

\section{CASIN treatment}

HSC were collected in serum-free expansion media and incubated at $37^{\circ} \mathrm{C}, 3 \%$ oxygen for 1 hour. Cells were then transferred into media \pm CASIN, incubated for 4 hours and washed. Cells were then used in subsequent experiments.

\section{Immunofluorescent staining}

Cells were seeded in serum-free expansion media, fixed and polarity for $\mathrm{Cdc} 42$ or tubulin assessed as previously described by Florian et al. ${ }^{9} \mathrm{Cdc} 42-\mathrm{GTP}$ in HSC was determined using the antibody described by Althoff et al. ${ }^{22}$ (see the Online Supplementary Methods for further details).

\section{Western blot}

A Rac/Cdc42 assay reagent (\# 14-325, Millipore) was used in pull down assays according to the manufacturer's protocol (see the Online Supplementary Methods for further details).

\section{Statistical analysis}

Statistical analyses were performed with GraphPad Prism 8 (version 8.1.2) and are presented as mean \pm standard deviation (SD) or mean \pm standard error of the mean (SEM) and box plots as minimum and maximum points. Kendall's correlation analysis was performed with $\mathrm{R}$ version 4.0.3, RStudio Team (2020) version 1.3.1093. ${ }^{*} P<0.05,{ }^{* *} P<0.005,{ }^{* * *} P<0.0005$.

\section{Results}

\section{Changes in the immunophenotypic frequencies of hematopoietic populations occur with age}

Data on whether there are changes in the frequency of HSC in the BM of humans upon aging remains controversial. This might be, at least in part, due to different gating strategies employed to identify human HSC. ${ }^{15,16}$ Using a more recently established and improved marker profile for the identification of human $\mathrm{HSC}^{21}$ (Online Supplementary Figure S1A), we first determined the frequency of HSC (Lin-CD34+ ${ }^{\text {sc low }}$ CD38-CD90+CD45ra-), HSPC (Lin-CD34+ ${ }^{\text {Ssc low }}$ CD38-) and Lin-CD34+ ${ }^{\text {ssc low }}$ cells in BM cells from the sternum of the elderly. While the frequency of the Lin-CD34+ ${ }^{\text {ssclow }}$ population did not change with age within our cohort, the HSPC frequency within Lin-CD34+ ${ }^{\text {ssc low }}$ population and HSC frequency within HSPC population increased with age (Figure $1 \mathrm{~A}$ to $\mathrm{C}$ ). 
A

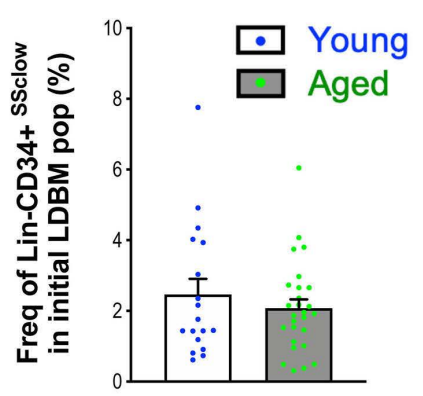

B

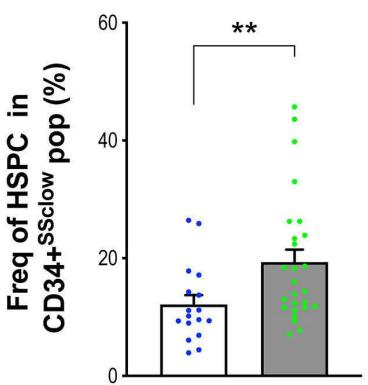

C

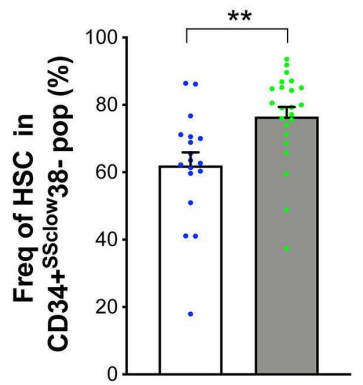

D

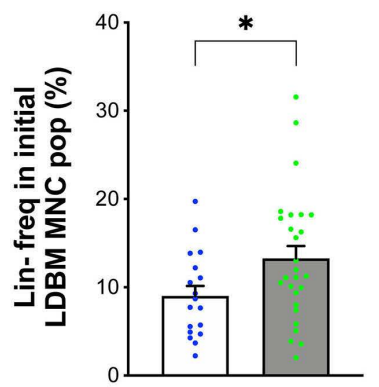

E
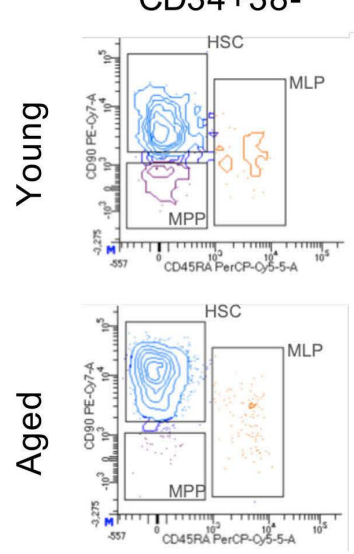

CD34+38+
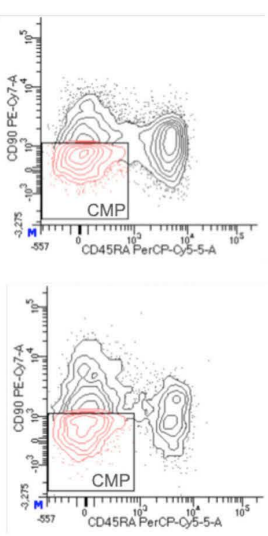

$\mathrm{F}$

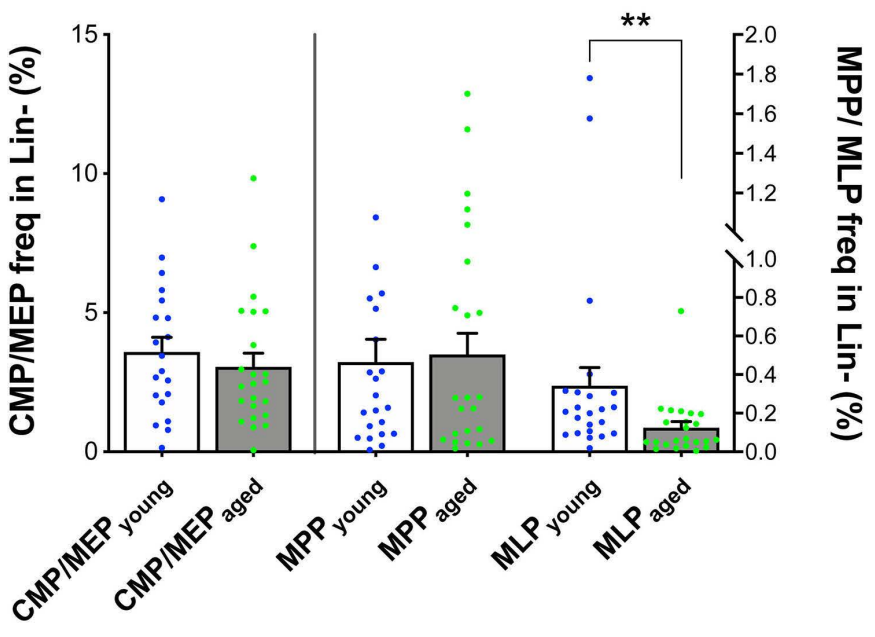

Figure 1. Flow cytometric analysis of different bone marrow populations isolated from young (white) and aged (grey) donors. Populations of interest within the lowdensity mononuclear cell fraction (MNC) were identified and frequencies of (A) Lin-CD34+ssc low, (B) hematopoietic stem progenitor cells (HSPC), (C) hematopoietic stem cells (HSC) and (D) Lin- cells from young and aged donors were determined. ${ }^{*} P<0.04, * * P<0.005$; Mann-Whitney and $t$-test with Welch's correction. Bars represent the mean \pm standard error of the mean (SEM). (E) Representative image of common myeloid progenitor/ megakaryocyte-erythroid progenitor (CMP/MEP), multipotent progenitor (MPP) and multipotent lymphoid progenitor (MLP) gates. (F) The frequency of CMP/MEP, MPP and MLP in the Lin- fraction of donors. $* * P=0.003$; Mann-Whitney test. Bars represent the mean \pm SEM. $17<\mathrm{n}_{\text {surs }}>20 ; 23<\mathrm{n}_{\text {sees }}>29$. Donor age: young $=23-39$ years $(\mathrm{yr})$, median $=27 \mathrm{yr} ;$ aged $=58-82 \mathrm{yr}$, median $=65 \mathrm{yr}$. $\mathrm{n}_{\text {sowe }}$ : number of young donors; $\mathrm{n}_{\text {sasa: }}$ number of aged donors.

These results support an increase in the HSPC population with age previously described for the iliac crest. ${ }^{16,23}$ Our observations further indicate that the age-related increase in frequency is not restricted to a single anatomical site or, in our studies, influenced by sex (Online Supplementary Figure $S 1 B$ and $C$ ). In addition, the frequency of HSC within the Lin-CD34 $+{ }^{\text {Ssc low }}$ population in the sternum was also significantly higher than in the young ${ }^{16}$ (Online Supplementary Figure S1D). We also found a not yet described increase in the frequency of lineage negative cells within the MNC population in aged donors (Figure 1D). The frequency of common myeloid progenitor/ megakaryocyte-erythroid progenitor (CMP/MEP, LinCD34+ ${ }^{\text {SSc low }}$ CD38+CD90-CD45ra-) and multipotent progenitor (MPP, Lin-CD34+ ${ }^{\text {Ssc low }}$ CD38-CD90-CD45ra-) did not change upon aging, while the frequency of immunophenotypic multipotent lymphoid progenitor (MLP, Lin-CD34+ ${ }^{\text {Scclow }}$ CD38-CD90-/low CD45ra+) within the Lin- population decreased significantly (Figure 1E and F). Our results demonstrate and confirm that aging is associated with an increase in the frequency of hematopoietic progenitor and HSC, but with a decrease in the frequency of MLP.

\section{Aged hematopoietic stem cells are delayed in the initiation of division}

We next tested whether the age-related increase in the HSC frequency might be linked to an elevated division rate of aged HSC. To this end, we determined the dynamics of first or second divisions of individual HSC ex vivo (Figure 2A). In general, BM-derived HSC showed a delayed initiation of division when compared to HSC from cord blood (CB) or HSC mobilized to blood (Online Supplementary Figure S2A and B). Surprisingly, aged HSC actually showed a delay until the first $50 \%$ of HSC underwent their first division compared to young HSC (Online Supplementary Figure S2B and C). This delay in initiation of division of aged HSC was still imminent in the presence of a different combination of cytokines (Online Supplementary Figure S2C to E) as well as under normoxic conditions (Online Supplementary Figure S2 F and G). The overall rate of division after initiation though was similar for both young and aged HSC for both the first division (Figure 2E) as well as the second division (Figure 2D to E; Online Supplementary Figure S2E). We next examined the proportion of cells in the distinct phases of the cell cycle by staining for DNA (Hoechst 33342) and Ki-67 (Ki-67 antibody) 
A

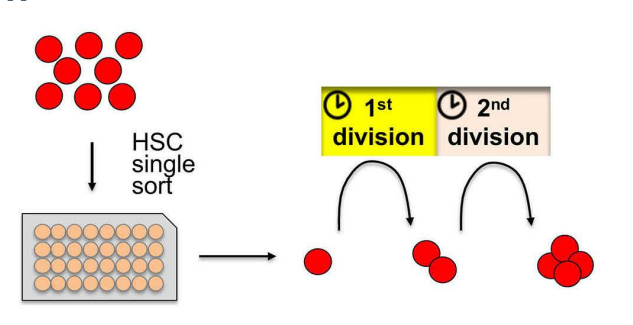

D

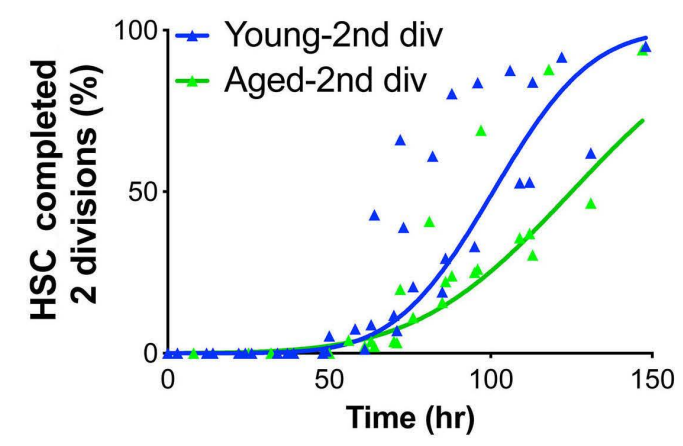

B

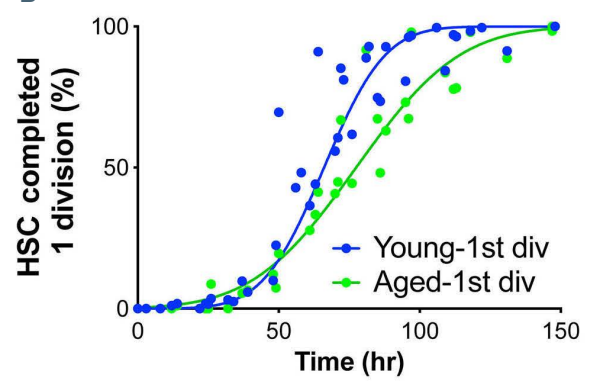

C

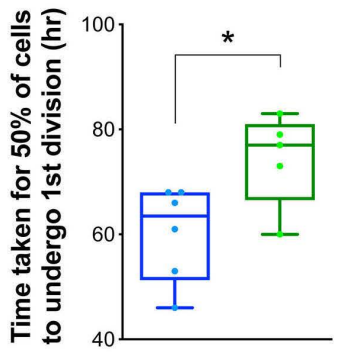

E

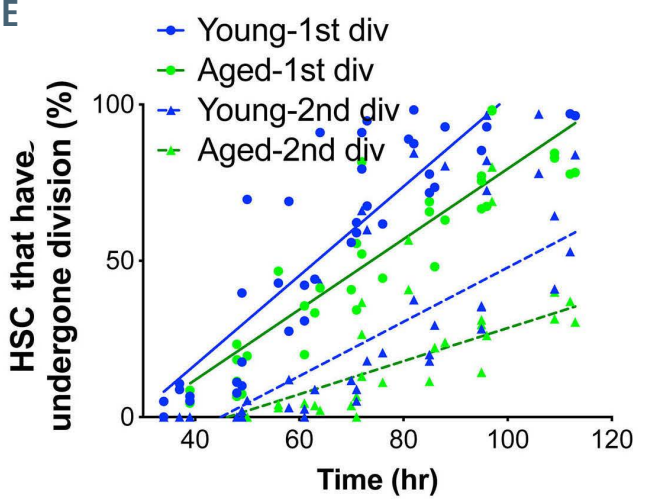

$$
\begin{aligned}
& \mathrm{M} 4 \\
& \mathrm{D} 1(\mathrm{~A}-\mathrm{Y})_{\text {slope }}=1.427 \pm 0.537 \mathrm{n} 39 ; P=0.661 \\
& \mathrm{D} 2(\mathrm{~A}-\mathrm{Y})_{\text {slope }}=0.5296 \pm 0.2387 \mathrm{n39} ; P=0.457 \\
& D\left(\mathrm{~A}_{1}-\mathrm{A}_{2}\right)_{\text {slope }}=1.126 \pm 0.422 \mathrm{n35} ; P=0.222 \\
& D\left(\mathrm{Y}_{1}-\mathrm{Y}_{2}\right)_{\text {slope }}=0.8686 \pm 0.3853 \mathrm{n} 43 ; P=0.401
\end{aligned}
$$

Figure 2. Kinetics of single cell division of young (blue) and aged (green) hematopoietic stem cells cultured ex vivo. (A) Experimental design of cells singly sorted into plates containing M4 media and incubated at $37^{\circ} \mathrm{C}, 3 \%$ oxygen. (B) Cumulative first division of live young and aged hematopoietic stem cells (HSC) and (C) time by which $50 \%$ of the cells have undergone the first division. ${ }^{*} P=0.03$; $t$-test with Welch's correction. Bars represent the mean \pm standard error the mean (SEM). (D) Cumulative second division of live young and aged HSC. (E) Slope was derived as linear regression fits and probability values calculated from the correlation coefficients (M4 calculations in the box). Each curve was derived from cumulative gaussian fits with robust regression. $n_{\text {,ome }}=6 ; n_{\mathrm{asea}}=5$ different donors. Donor age: young $=27-39$ years $(\mathrm{yr})$, median $=28 \mathrm{yr}$; aged $=64-75 \mathrm{yr}$, median $=69 \mathrm{yr}$.

(Online Supplementary Figure S3A and B). The proportions of young and aged HSC in the $G_{0}, G_{1}$ and $S-G_{2}-M$ phases of the cell cycle were similar, hence the delayed initiation of division was not simply driven by a higher frequency of quiescent cells upon aging. In aggregation, aged HSC show a delayed initiation of division that is not linked to a higher frequency of cells in $\mathrm{G}_{0}$ or $\mathrm{G}_{\mathrm{t}}$, while once division is initiated, there is no difference in the overall kinetics of the division.

Cdc42 activity is increased in human hematopoietic stem cells from the elderly and correlates with an increase in hematopoietic stem cell frequency

The small RhoGPTase Cdc42 cycles between an active (GTP bound) and inactive (GDP bound) state ${ }^{24}$ and has been shown to have essential roles in HSC regulation. ${ }^{25}$ We previously demonstrated that the active form (GTPbound form) of the small RhoGTPase Cdc42 was increased in murine low-density bone marrow (LDBM) cells as well as in HSC upon aging, and that this increase in HSC resulted in the age-related increase in HSC frequency in mice. ${ }^{9}$ We therefore determined the level of activity of Cdc42 in human LDBM from the elderly by a standard pull down assay (Figure 3A; Online Supplementary Figure S4A). Pull-down replicates on the same samples from randomized donors demonstrated overall high reproducibility of the pull-down assay also on primary human samples (Online Supplementary Figure S4B). We observed an approximate 4-fold increase in the relative Cdc42 activity in LDBM cells from elderly donors compared to the young (Figure $3 \mathrm{~B}$ ) which was re-affirmed in correlation analysis of $\mathrm{Cdc} 42$ activity and age (Online Supplementary Figure S4C). Using immunofluorescence imaging, we found aged HSC had significantly higher Cdc42-GTP expression than young HSC (Figure 3C to E; Online Supplementary Figure S4D), implying primitive stem cells also undergo Cdc42 activity changes upon aging. In addition, we identified a positive association between Cdc42 activity and HSC frequency but not between Cdc42 activity and HSPC frequency (Figure 3F and G). Furthermore, the spread of values and, thus, the standard deviation (Figure $3 \mathrm{~B}$ ) was higher in the aged group than in the young, which points to a strong increase in heterogeneity of the hematopoietic system in individuals upon aging. Heterogeneity upon aging in mice is, for example, less pronounced due to their inbred nature. ${ }^{26-28}$ In aggrega- 
A
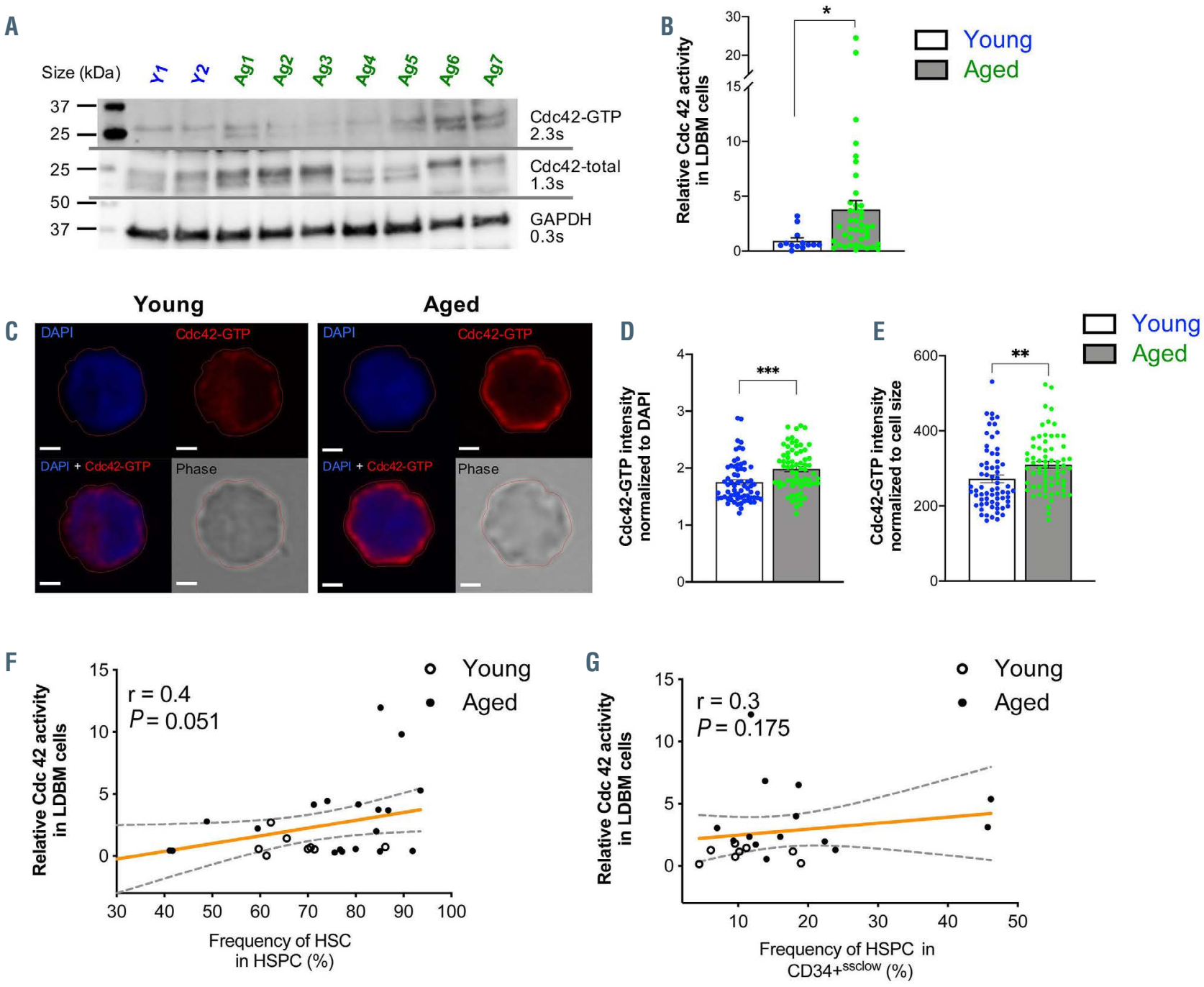

Figure 3. Relative expression of Cdc42 activity in young and aged hematopoietic stem cells. (A) Representative image of western blot. (B) Quantitative expression of relative Cdc42 activity normalized to glyceraldehyde 3-phosphate dehydrogenase (GAPDH). *P=0.010, if the two points with activity higher than 20 are excluded, then $P=0.017$; Mann-Whitney test. Bars represent the mean \pm standard error of the mean (SEM). $\mathrm{n}_{\text {san }}=13$; $\mathrm{n}_{\text {ase }}=41$. Donor age: young $=23-39$ years $(\mathrm{yr})$, median $=27$ $\mathrm{yr}$; aged $=60-82 \mathrm{yr}$, median $=66 \mathrm{yr}$. (C) Representative confocal image of Cdc42-GTP expression in hematopoietic stem cells (HSC). Cdc42-GTP quantification of young and aged HSC normalized to (D) DAPI intensity and (E) cell size. $* * * P=0.0001, * * P=0.0012$; Mann-Whitney test. Bars $=$ mean \pm SEM and $n_{\text {sems }}=66$; $n_{\text {nase }}=67$, from three different donors per cohort. Donor age: young $=27-31$ years (yr), median $=27 \mathrm{yr}$; aged $=63-76 \mathrm{yr}$, median $=76 \mathrm{yr}$. Scale bar represents $2 \mu \mathrm{m}$. $(\mathrm{F})$ Correlation analysis (Spearman) of relative Cdc42 activity and HSC frequency $(r=0.4, P=0.05, n=37)$ and $(G)$ hematopoietic stem progenitor cell (HSPC) frequency ( $r=0.3$, $P=0.175, n=22)$, broken grey lines represent 95\% Confidence Interval.

tion, the data show that similar to mice, primitive hematopoietic cells from elderly humans show elevated Cdc42 activity. The level of Cdc42 activity in individuals correlates positively with the frequency of HSC, supporting a possible role for $\mathrm{Cdc} 42$ activity in causing the elevated HSC frequency, similar to what has been described in the mouse. ${ }^{8,29}$

\section{The frequency of hematopoietic stem cells polar for Cdc42 and tubulin declines upon aging}

Another established age-related hallmark for murine HSC is the reduction in frequency of cells polar for cytosolic polarity proteins like tubulin and Cdc42 (Figure 4A). This "apolarity" of aged murine HSC is a direct consequence of the elevated activity of Cdc42 itself in aged HSC ${ }^{9}$ and likely results in a change in the mode of the division of aged murine HSC. ${ }^{8}$ We therefore determined the frequency of aged human HSC that showed a polar distribution of
Cdc42 and tubulin by immunofluorescence analyses (Figure $4 \mathrm{~B}$ to D). Approximately $70 \%$ of young HSC showed a polar distribution of $\mathrm{Cdc} 42$ while approximately $70 \%$ of the aged HSC showed an apolar distribution (Figure 4C). The frequency of aged HSC polar for tubulin was also reduced (Figure 4D). Our findings establish that aged human HSC present with a reduced frequency of cells polar for polarity proteins. Oddly, we did not find a significant association between $\mathrm{Cdc} 42$ polarity and tubulin polarity in human HSC (Online Supplementary Figure S5A) suggesting these two parameters might not be directly correlated as has been shown for murine HSC. ${ }^{9}$ Nonetheless, we observed a strong negative association between Cdc42 polarity and age (Online Supplementary Figure S5B), implying the frequency of cells that remain polar decreases with increasing age. In order to determine whether the level of Cdc42 activity in human cells might be linked to the frequency of cells polar for $\mathrm{Cdc} 42$, linear regression analyses 
of Cdc42 activity and Cdc42 polarity were performed on human HSC. The data revealed that indeed Cdc 42 activity and the frequency of polar HSC were inversely correlated (Figure 4E), suggesting a causative link between Cdc42 activity and polarity also for human HSC.

\section{Aged hematopoietic stem cells are repolarized by pharmacological inhibition of Cdc42 activity}

CASIN (Cdc42 activity specific inhibitor) is a pharmacological compound that specifically inhibits the activity of Cdc42. ${ }^{30,31}$ We thus tested whether inhibition of Cdc42 activity in aged human HSC via CASIN might result in an increase in the frequency of polar HSC. CASIN indeed increased the frequency of HSC polar for Cdc42 in a dose-dependent fashion (Figure 4F) but not for tubulin (Online Supplementary Figure S5C). Inhibition of Cdc42 activity, thus, increases the frequency of aged HSC polar for Cdc42, though not fully to the frequency seen in young HSC (Figure $4 \mathrm{C}$ ). This data confirms that elevated activity of Cdc42 is, at least in part, causative for the reduced polarity of aged human HSC similar to aged murine HSC. ${ }^{8,9,32}$

\section{CASIN treatment induces early division onset in aged hematopoietic stem cells}

We previously demonstrated that HSC from aged donors were delayed in division onset in comparison to HSC isolated from the young (Figure $2 \mathrm{~B}$ and $\mathrm{C}$ ), hence we assessed the effect CASIN treatment would have on the division of aged HSC. Our results show that upon CASIN treatment, aged HSC commence division hours before untreated HSC (Figure 4G) with no additional effect on the second division (Online Supplementary Figure S6D to E). We observed that the time taken for $50 \%$ of untreated
A

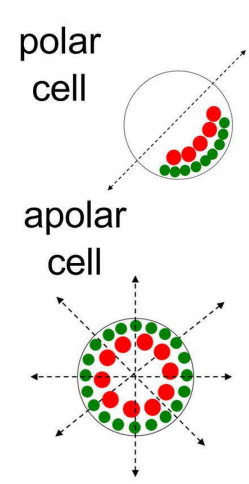

C

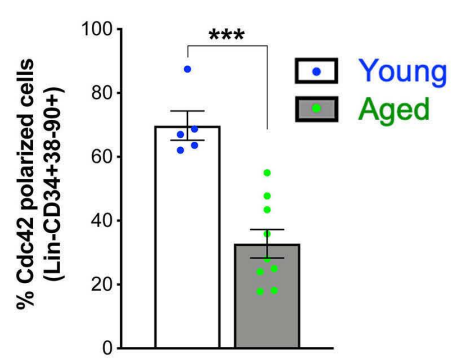

D

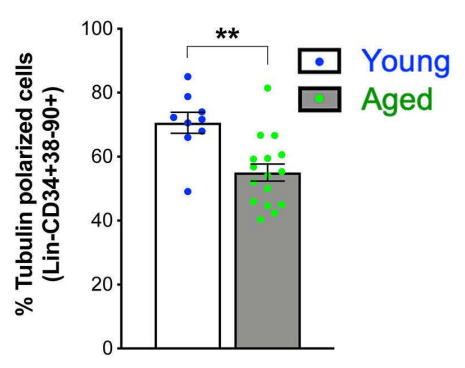

B
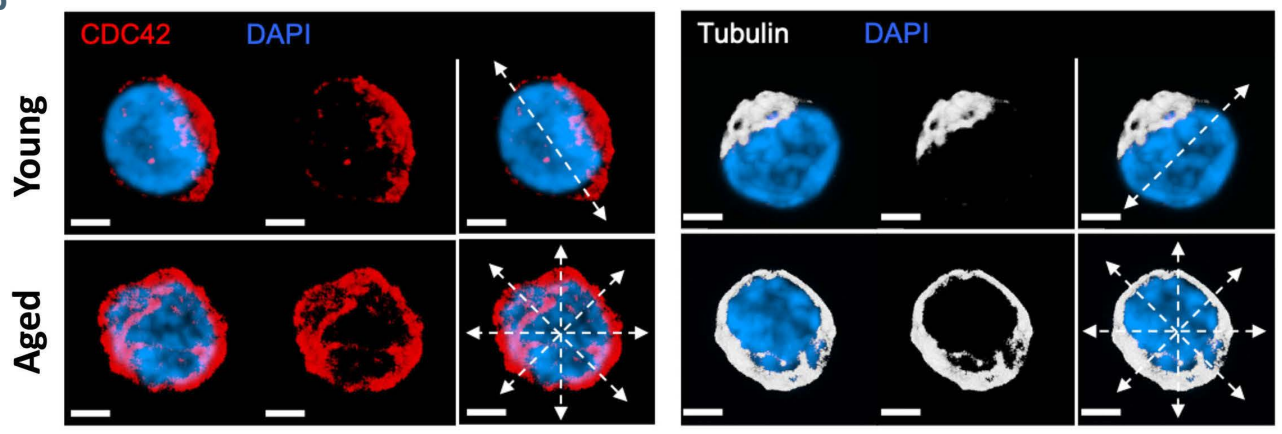

$E$

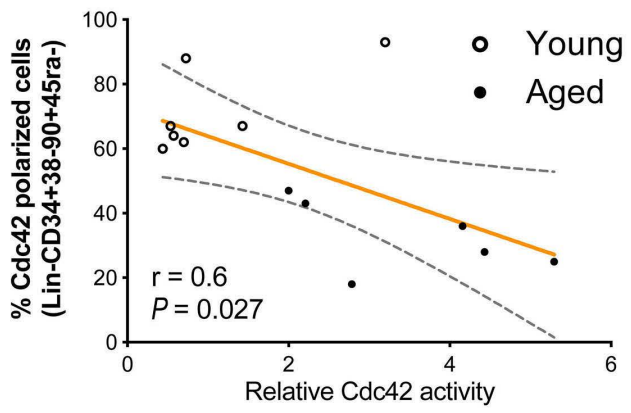

$\mathrm{H}$

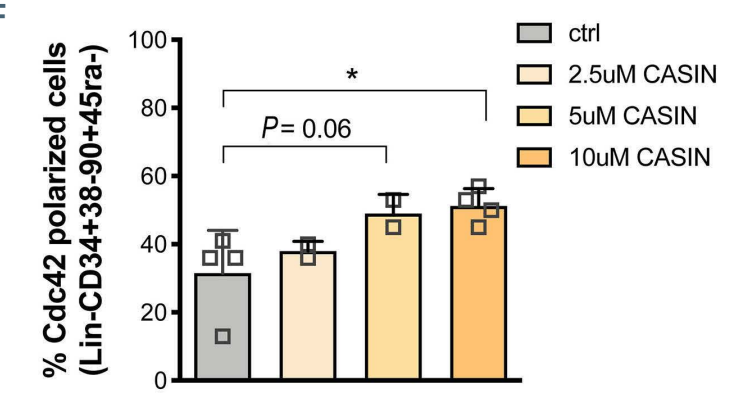

G
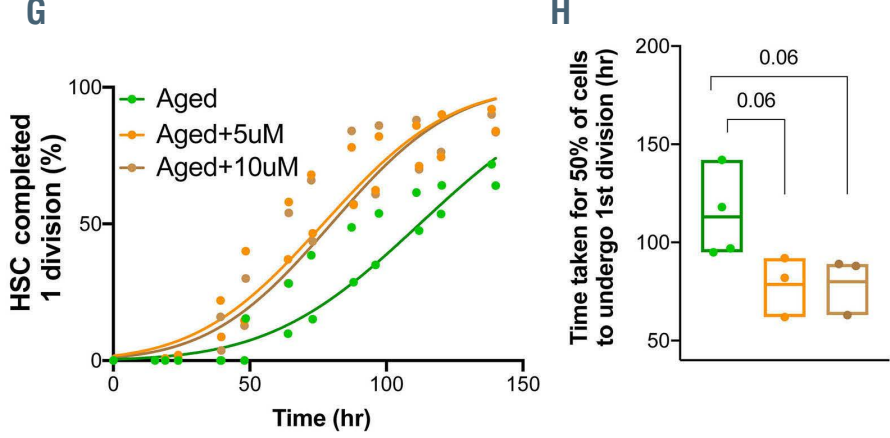

Figure 4. Hematopoietic stem cells polarity assessment and division kinetics in the presence and absence of CASIN. (A) Illustration of a polar and apolar cell. (B) Representative immunofluorescent images taken with a confocal microscope. Quantification of the proportion of young (white) and aged (grey) donor cells polar for (C) Cdc42 and (D) tubulin. $* * * P=0.00018, * * P=0.002$; $t$-test, false discovery rate approach. Bars represent the mean \pm standard error of the mean (SEM). Scale bar represents $2 \mu \mathrm{m} ; \mathrm{n}_{\text {,uns }}>5$; $\mathrm{n}_{\text {sex }}>8$. Donor age: young $=26-39$ years (yr), median $=28 \mathrm{yr}$; aged $=58-82 \mathrm{yr}$, median $=65 \mathrm{yr}$. (E) Correlation analysis (Pearson) of Cdc42 polarity and relative Cdc42 activity ( $r=0.6, P=0.03, n=13)$, broken grey lines represent $95 \%$ Confidence Interval. (F) Quantification of Cdc42 polarity of hematopoietic stem cells (HSC) after treatment with CASIN. ${ }^{*} P=0.03$; Mann-Whitney test. Bars represen the mean \pm SD. $\mathrm{n}_{\text {mes }}>2$. Donor age: aged $=61-81$ years (yr), median $=62$ yr. (G) Cumulative first division of aged HSC with and without CASIN and $(\mathrm{H})$ time by which $50 \%$ of the cells have undergone the first division. Curve was derived from cumulative gaussian fits with robust regression. $n_{\text {aese }}$ and $n_{\text {aesectises }}=4$. Donor age: aged $=63-71 \mathrm{yr}$, median $=66 \mathrm{yr}$. 
HSC to undergo first division was considerably longer than cells treated with $5 \mu \mathrm{M}$ or $10 \mu \mathrm{M}$ CASIN albeit not statistically significant $(P=0.06$, Figure $4 \mathrm{H})$ suggesting the inhibition of Cdc42 activity may facilitate or support early division onset.

\section{Aged hematopoietic stem cells show an elevated level of chimerism but balanced differentiation in NBSGW xenotransplants}

Murine xenotransplant models that are used to test the potential of human HSC usually require irradiation of the recipient animal for establishing human engraftment. ${ }^{17}$ Irradiation of the recipients though might contribute to variable secondary effects not linked to the potential of the transplanted HSC. In order to circumvent the necessity for irradiation of the recipients in xenotransplants, we utilized NBSGW animals as recipients ${ }^{33}$ to assess the function of young, aged and CASIN-treated aged human HSC in vivo.

To this end, 500 HSC were injected via the tail vein into mice and BM chimerism was analyzed at week 8 and 12 post xenotransplantation. Mice were considered to be successfully engrafted when the level of human hematopoietic cells (huCD45+) detected was higher than $0.1 \%$ of total CD45+ cells. ${ }^{34}$ In general, transplants with human BM- derived HSC result in a much lower level of chimerism in comparison to transplants with CB-derived HSC (data not shown). Animals transplanted with aged human HSC showed a significantly higher level of engraftment (mean $3.7 \%$ ) compared to recipients that received young HSC (mean $0.6 \%$ ) and the aged + CASIN groups (mean 1.6\%, $2.1 \%$ ) (Figure $5 \mathrm{~A}$ ). Chimerism driven by aged HSC further increased at 12 weeks post-transplantation (mean 5\%) compared to chimerism driven by young HSC (Figure 5B). This result was surprising, as the small number of previous studies in which aged human cells were transplanted into irradiated recipients demonstrated a lower ${ }^{16}$ or at least similar chimerism ${ }^{15}$ stemming from aged compared to young human HSC. In order to test whether irradiation of recipients might influence the reconstitution potential of aged HSC, we also transplanted young and aged human HSC into irradiated (1.6 Gy) NBSGW mice. Recipients of aged HSC displayed similar levels of chimerism compared to recipients of young HSC, and only marginal differences in their differentiation profile (Online Supplementary Figure $S 6 A$ to C). Irradiation of recipients might thus influence the reconstitution potential of aged human HSC in xenotransplantation experiments. In summary, our results demonstrate that aged HSC show an overall elevated contribution to BM upon transplantation into NBSGW animals.
A

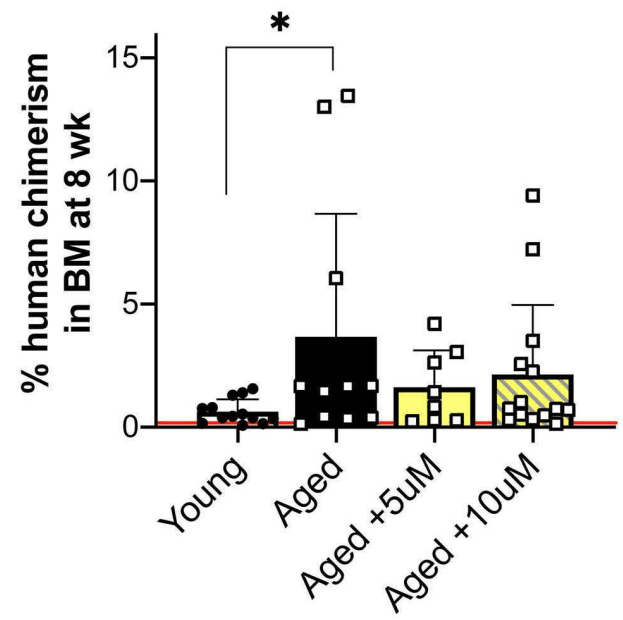

C

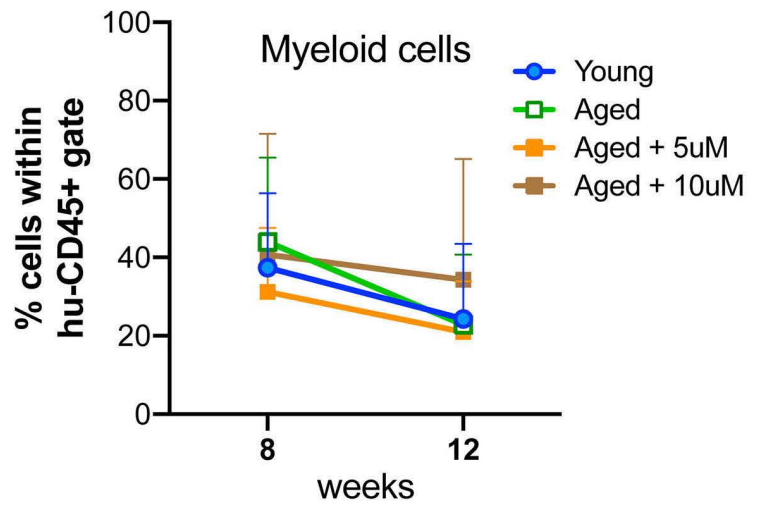

B

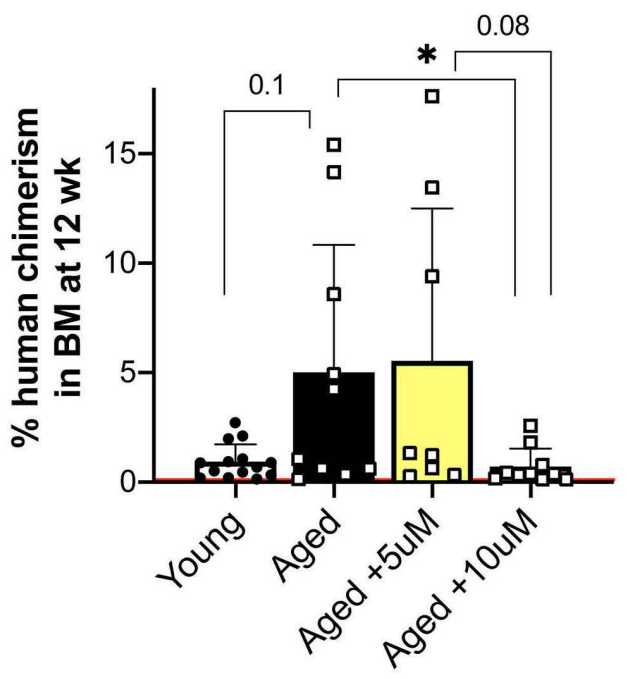

D

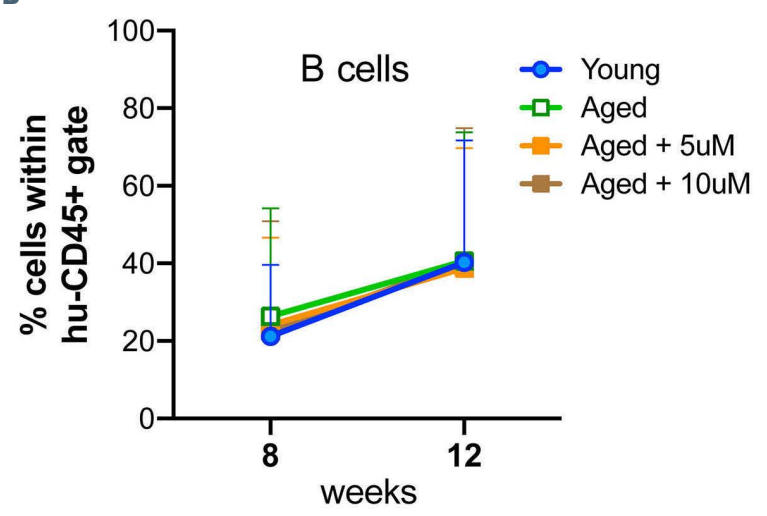

Figure 5. Analysis of xenotransplantation experiment under non-irradiated settings. Frequency of human cells in the bone marrow (BM) of NBSGW mice at (A) 8 weeks and (B) 12 weeks post-transplant. (C) Emergence of human myeloid cells and (D) B cells post-transplant from young (blue), aged (green) and CASIN-treated aged (orange, brown) -recipients. Red line represents averages of non-transplanted controls. Bars represent the mean " \pm " standard deviation $(S D)$. $n=6$ different donors per cohort, 46 mice at 8 weeks and 42 mice at 12 weeks. Donor age: young =24-35 years (yr), median =27 yr; aged =60-81 yr, median =62 yr. 
Aged HSC treated with CASIN at $5 \mu \mathrm{M}$ or $10 \mu \mathrm{M}$ were more similar in their contribution to chimerism to young HSC at 8 weeks post transplantation. Aged HSC treated with the higher concentration of CASIN, $10 \mu \mathrm{M}$, were able to sustain a significantly lower, young-like contribution to chimerism at 12 weeks post transplantation. This mirrors our finding that aged HSC, treated with $10 \mu \mathrm{M}$ of CASIN, showed the highest increase in the repolarization of aged HSC (Figure 4F). Assessment of the frequency of human myeloid (CD33+) and B cells (CD19+) in the BM of recipient mice revealed no significant differences among any of the experimental groups (Figure 5C and D) implying that aged human HSC, at least in NBSGW animals, show no differentiation skewing as reported for aged murine HSC.

Colony forming unit assays also revealed the myeloerythroid potential of aged and CASIN-treated aged HSC were preserved (Online Supplementary Figure S6F to $H$ ) which is in line with the report by Pang et al. ${ }^{16}$

In summary, our xenotransplantation experiments demonstrate that, to our surprise, aged human HSC are able to confer an elevated level of chimerism upon transplantation into NBSGW animals, which is likely a result of the elevated activity of Cdc42 in aged HSC, as aged HSC treated with $10 \mu \mathrm{M}$ of CASIN are more similar to young HSC than to aged HSC.

\section{Discussion}

In this study, we characterized aging-related changes in human HSC and tested an intervention to target these aging-related changes. Using a well-established marker panel for the identification of primitive hematopoietic cells in BM, we show that there is an increase in the HSC frequency and a decrease in lymphoid progenitors in BM of the sternum of the elderly. We specifically selected the low side scatter CD34+ cells as our reference population in accordance to the guidelines of the International Society of Hematotherapy and Graft Engineering (ISHAGE). ${ }^{35}$ Additionally, the gating of low side scatter CD34+ cells minimizes variation across labs thereby increasing reproducibility and reliability. ${ }^{35}$ It is a possibility that the disparities in previous reports on changes in the frequency of human $\mathrm{HSC}^{15,16}$ may result from the use of different reference populations. Our data also demonstrates a delayed entry of aged HSC into division, an observation similarly made by Flach but not us for aged murine HSC, $, 3,36$ but no overall delay in division kinetics once the cells start to divide. This finding is in contrast to observations by Pang et $a l .{ }^{16}$ which concluded that aged HSC are more prone to divide. Here, Pang determined the proportion of $G_{0}$ and non-G cells using Hoechst 33342 and Pyronin $Y$ while we used Hoechst 33342 and Ki-67. While Pyronin Y correlates with RNA content, Ki-67 is seen as a marker for proliferation in itself. It is thus a possibility that upon aging the correlation between elevated RNA content and proliferation might be somewhat diminished. The molecular mechanisms of the delayed entry of aged human HSC into cell division upon stimulation might be linked to elevated Cdc42 activity as cells treated with a pharmacological inhibitor of Cdc42 activity (CASIN), proceeded into division ahead of their untreated counterparts. In mutant yeast, hyperactive levels of $\mathrm{Cdc} 42$ result in the formation of multiple budding sites, however in wild- type cells, foci competition is quickly resolved, with only one axis maturing and recruiting other proteins such as Bem1, to facilitate division. ${ }^{37}$ It is therefore a possibility that also in aged human HSC, the correct allocation of proteins to distinct positions within cells that are critical for proper initiation of division, might be altered upon elevated activity of Cdc42, resulting in a delay.

Knowledge on the molecular mechanisms underlying aging of HSC have supported the development of therapeutic approaches to mitigate aging of HSC. For example, CASIN shows great promise in rejuvenating aged $\mathrm{HSC}^{9}$ and also in reverting aging-associated immune remodeling (AAIR) in murine models. ${ }^{32}$ A multivariate analysis model of gene expression profiles and biological age identified Cdc42 as a strong predictor of survival and that a higher Cdc42 level is associated with higher mortality. ${ }^{38}$ We showed previously that the level of Cdc42 activity in human blood cells correlates positively with age ${ }^{39}$ and in this study, we identified a similar association in LDBM cells. Aged human HSC also show elevated activity of Cdc42 and a low frequency of HSC polar for polarity proteins. We also report a negative correlation between the activity of $\mathrm{Cdc} 42$ in individual donors and the frequency of HSC polar for polarity proteins like Cdc42 in that donor. We could demonstrate that the inhibition of the activity of Cdc42 is sufficient to increase the frequency of chronologically aged HSC polar for Cdc42 but not for tubulin which suggests in human cells, Cdc42 and tubulin axes may not be closely linked as shown in murine cells. Furthermore, the number of HSC in a given donor and the activity of Cdc42 in hematopoietic cells of that donor are positively correlated, which suggests that $\mathrm{Cdc} 42$ activity may affect the frequency of human HSC upon aging and could therefore, directly or indirectly, contribute to the increased HSC frequency observed. Given that in mice, apolar distribution of Cdc42 (a consequence of elevated activity of Cdc42) drives HSC to divide symmetrically, ${ }^{8}$ and that elevated activity of Cdc42 in human leukemia stem cells is linked to more symmetric divisions, ${ }^{40}$ we postulate here an increased frequency of symmetric divisions also for HSC of the elderly, which though will require further investigations.

In order to assess changes in function upon aging, HSC were transplanted into NBSGW recipients. By employing this model, secondary elements that may be introduced due to irradiation were avoided. ${ }^{41}$ We noted higher chimerism levels in non-conditioned recipients of aged HSC whereas conditioned recipients showed no increase. These observations also corroborate findings that irradiation, even at low doses, induces functional changes in mesenchymal stromal cells which influences their hematopoietic-supporting ability. ${ }^{42}$ Our data suggests that the elevated Cdc42 activity in aged HSC drives the age-related high level of chimerism observed in non-irradiated NBSGW recipients since recipients of young and $10 \mu \mathrm{M}$ CASINtreated aged HSC exhibited close similarities in the level of human chimerism. The higher level of chimerism driven by aged human HSC further supports a model in which human aged HSC might predominantly undergo symmetrical divisions. Another possibility remains that an elevated repopulation potential of aged human HSC could be driven by the presence of individual clones ${ }^{43}$ linked to aging-related clonal hematopoiesis reported for a certain percentage of the elderly. ${ }^{44}$ Most human studies though demonstrated clonal hematopoiesis only in peripheral blood and not 
among HSC, thus it remains unclear whether changes in clonality exist already among aged HSC. ${ }^{446}$ We did not determine in this study clonality among the small number of HSC transplanted into animals, as this remains technically very challenging.

In summary, we identified novel age-related phenotypes of human HSC and provide evidence of inter-species parallels as well as differences to support translational studies in the aging field. Our data supports that age-related phenotypes that are indicators of the function of aged HSC (Cdc42 activity, polarity, reconstitution potential in xenografts) are malleable in human HSC by inhibition of the age-related elevated activity of $\mathrm{Cdc} 42$. This might therefore present a new possibility to improve autologous stem cell transplants of aged donors.

\section{Disclosures}

No conflicts of interest to disclose.

\section{Contributions}

$A A, H G$ was involved in study design, interpretation and manuscript writing; AA performed and analyzed experiments;
$R E, M K$ and $A L$ provided aged samples; $A K$ and $K S$ performed experiments; KS, $A V$ and $K E$ assisted in cell sorting procedures; VS supported in transplantation and bone marrow aspiration; YZ and MCF assisted in study design; $A L, R E$, $M K$ and MCF reviewed and edited the manuscript.

\section{Acknowledgements}

We would like to thank the Flow Cytometry Core and Imaging Core Facilities and the Tierforschungszentrum at the University of UIm for their support. We also thank Jeffrey Bailey for excellent training in the bone marrow aspiration technique, Aishlin Hassan and Kalpana Nattamai for their technical support at Cincinnati Children's Hospital Medical Center (CCHMC).

\section{Funding}

This study was supported with funding from the DFG, GRK1789 (CEMMA) to HG and AA.

\section{Data sharing statemen}

The data that support the findings of this study are available from the corresponding author upon reasonable request.

\section{References}

1. Kovtonyuk L V., Fritsch K, Feng X, Manz MG, Takizawa $H$. Inflamm-aging of hematopoiesis, hematopoietic stem cells, and the bone marrow microenvironment. Front Immunol. 2016;7:502.

2. Ponnappan S, Ponnappan U. Aging and immune function: molecular mechanisms to interventions. Antioxid Redox Signal. 2011;14(8):1551-1585.

3. De Haan G, Lazare SS. Aging of hematopoietic stem cells. Blood. 2018;131(5):479-487.

4. Geiger $\mathrm{H}$, de Haan G, Florian MC. The ageing haematopoietic stem cell compartment. Nat Rev Immunol. 2013;13(5):376-389.

5. Chambers SM, Shaw CA, Gatza C, Fisk CJ, Donehower LA, Goodell MA. Aging hematopoietic stem cells decline in function and exhibit epigenetic dysregulation. PLoS Biol. 2007;5(8):1750-1762.

6. Liang Y, Van Zant G, Szilvassy SJ. Effects of aging on the homing and engraftment of murine hematopoietic stem and progenitor cells. Blood. 2005;106(4):1479-1487.

7. Beerman I, Seita J, Inlay MA, Weissman IL, Rossi DJ. Quiescent hematopoietic stem cells accumulate DNA damage during aging that is repaired upon entry into cell cycle. Cell Stem Cell. 2014;15(1):37-50.

8. Florian MC, Klose M, Sacma M, et al. Aging alters the epigenetic asymmetry of HSC division. PLoS Biol. 2018;16(9):1-35.

9. Florian MC, Dörr K, Niebel A, et al. Cdc42 activity regulates hematopoietic stem cell aging and rejuvenation. Cell Stem Cell. 2012;10(5):520-530.

10. Abkowitz JL, Golinelli D, Harrison DE, Guttorp P. In vivo kinetics of murine hemopoietic stem cells. Blood. 2000;96(10):33993405.

11. Catlin SN, Busque L, Gale RE, Guttorp P, Abkowitz JL. The replication rate of human hematopoietic stem cells in vivo. Blood. 2011;117(17):4460-4466.

12. Lee CCI, Fletcher MD, Tarantal AF. Effect of age on the frequency, cell cycle, and lineage maturation of rhesus monkey (Macaca mulatta) CD34+and hematopoietic progenitor cells. Pediatr Res. 2005;58(2):315-322.
13. Geiger H, Rennebeck G, Van Zant G. Regulation of hematopoietic stem cell aging in vivo by a distinct genetic element. Proc Natl Acad Sci. 2005;102(14):510-5107.

14. Geiger H, True JM, De Haan G, Van Zant G. Age- and stage-specific regulation patterns in the hematopoietic stem cell hierarchy. Blood. 2001;98(10):2966-2972.

15. Kuranda K, Vargaftig J, de la Rochere P, et al. Age-related changes in human hematopoietic stem/progenitor cells. Aging Cell. 2011;10(3):542-546.

16. Pang WW, Price EA, Sahoo D, et al. Human bone marrow hematopoietic stem cells are increased in frequency and myeloid-biased with age. Proc Natl Acad Sci U S A. 2011;108(50):20012-20017.

17. Doulatov S, Notta F, Laurenti E, Dick JE. Hematopoiesis: a human perspective. Cell Stem Cell. 2012;10(2):120-136.

18. Cosgun KN, Rahmig S, Mende N, et al. Kit regulates HSC engraftment across the human-mouse species barrier. Cell Stem Cell. 2014;15(2):227-238.

19. McIntosh BE, Brown ME, Duffin BM, et al. Nonirradiated NOD,B6.SCID Il2ry-/kitW41/W41(NBSGW) mice support multilineage engraftment of human hematopoietic cells. Stem Cell Rep. 2015;4(2):171-180.

20. Waskow C, Madan V, Bartels S, Costa C, Blasig R, Rodewald HR. Hematopoietic stem cell transplantation without irradiation. Nat Methods. 2009;6(4):267-269.

21. van Galen P, Kreso A, Wienholds E, et al. Reduced lymphoid lineage priming promotes human hematopoietic stem cell expansion. Cell Stem Cell. 2014;14(1):94106.

22. Althoff MJ, Nayak RC, Hegde S, et al. Yap1Scribble polarization is required for hematopoietic stem cell division and fate. Blood. 2020;136(16):1824-1836.

23. Beerman I, Maloney WJ, Weissmann IL, Rossi DJ. Stem cells and the aging hematopoietic system. Curr Opin Immunol. 2010;22(4):500-506.

24. Etienne-Manneville S, Hall A. Rho GTPases in cell biology. Nature. 2002;420(6916):629635.

25. Yang L, Zheng Y. Cdc42: A signal coordina- tor in hematopoietic stem cell maintenance. Cell Cycle. 2007;6(12):1444-1449.

26. Gekas C, Graf T. CD41 expression marks myeloid-biased adult hematopoietic stem cells and increases with age. Blood. 2013;121(22):4463-4472.

27. Glauche I, Thielecke L, Roeder I. Cellular aging leads to functional heterogeneity of hematopoietic stem cells: a modeling perspective. Aging Cell. 2011;10(3):457-465.

28. Akunuru S, Geiger H. Aging, clonality, and rejuvenation of hematopoietic stem cells. Trends Mol Med. 2016;22(8):701-712.

29. Klose M, Florian MC, Gerbaulet A, Geiger H, Glauche I. Hematopoietic stem cell dynamics are regulated by progenitor demand: lessons from a quantitative modeling approach Stem Cells. 2019;37(7):1-22.

30. Liu W, Du W, Shang X, et al. Rational identification of a $\mathrm{Cdc} 42$ inhibitor presents a new regimen for long-term hematopoietic stem cell mobilization. Leukemia. 2019;33(3):749 761.

31. Du W, Liu W, Mizukawa B, et al. A nonmyeloablative conditioning approach for long-term engraftment of human and mouse hematopoietic stem cells. Leukemia. 2018;32 (9):2041-2046.

32. Leins H, Mulaw M, Eiwen K, et al. Aged murine hematopoietic stem cells drive agingassociated immune remodeling. Blood. 2018;132(6):565-576.

33. McIntosh BE, Brown ME, Duffin BM, et al Nonirradiated NOD,B6.SCID Il2ry-/kitW41/W41 (NBSGW) mice support multilineage engraftment of human hematopoietic cells. Stem Cell Rep. 2015;4(2):171-180.

34. Martin MG, Welch JS, Uy GL, et al. Limited engraftment of low-risk myelodysplastic syndrome cells in NOD/SCID gamma-C chain knockout mice. Leukemia. 2010;24(9): 1662-1664

35. Sutherland DR, Anderson L, Keeney M Nayar R, Chin-Yee I. The ISHAGE guidelines for CD34+ cell determination by flow cytometry. J Hematother. 1996;5(3):213-226.

36. Flach J, Bakker ST, Mohrin $M$, et al. Replication stress is a potent driver of functional decline in ageing haematopoietic stem cells. Nature. 2014;512(7513):198-202. 
37. Witte K, Strickland D, Glotzer M. Cell cycle entry triggers a switch between two modes of Cdc42 activation during yeast polarization. Elife. 2017;6:e26722.

38. Kerber RA, O'Brien E, Cawthon RM. Gene expression profiles associated with aging and mortality in humans. Aging Cell. 2009;8(3):239-250.

39. Florian MC, Klenk J, Marka G, et al. Expression and activity of the small RhoGTPase Cdc42 in blood cells of older adults are associated with age and cardiovascular disease. J Gerontol Ser. A 2017;72(9):1196-1200.

40. Mizukawa B, O’Brien E, Moreira DC, et al.
The cell polarity determinant CDC42 controls division symmetry to block leukemia cell differentiation. Blood. 2017;130(11): 1336-1346.

41. Costa S, Reagan MR. Therapeutic irradiation: consequences for bone and bone marrow adipose tissue. Front Endocrinol (Lausanne). 2019;10:587.

42. Preciado S, Muntión S, Rico A, et al. Mesenchymal stromal cell irradiation interferes with the adipogenic/osteogenic differentiation balance and improves their hematopoietic-supporting ability. Biol Blood Marrow Transplant. 2018;24(3):443-451.

43. Belderbos ME, Jacobs S, Koster TK, et al.
Donor-to-donor heterogeneity in the clonal dynamics of transplanted human cord blood stem cells in murine xenografts. Biol Blood Marrow Transplant. 2020;26(1):16-25.

44. Jaiswal S, Natarajan P, Silver AJ, et al. Clonal hematopoiesis and risk of atherosclerotic cardiovascular disease. N Engl J Med. 2017;377 (2):111-121.

45. Jaiswal S, Fontanillas P, Flannick J, et al. Agerelated clonal hematopoiesis associated with adverse outcomes. N Engl J Med. 2014;371 (26):2488-2498

46. Sano S, Wang Y, Walsh K. Clonal hematopoiesis and its impact on cardiovascular disease. Circ J. 2019;83(1):2-11. 